\title{
SECAGEM DE PASTA ALIMENTÍCIA EM LEITO DE JORRO: AVALIAÇÃO DOS ASPECTOS ENERGÉTICOS E PRODUÇÃO
}

\author{
M. B. BRAGA ${ }^{1 *}$, S. C. S. ROCHA ${ }^{1}$ \\ ${ }^{1}$ Universidade Estadual de Campinas, Faculdade de Engenharia Química, Departamento de \\ Engenharia de Processos \\ *e-mail: matheus19braga@yahoo.com.br
}

\begin{abstract}
RESUMO
O principal objetivo deste trabalho foi analisar o processo de secagem da pasta leite reconstituído-polpa de amora $(25 \%: 75 \%(\mathrm{~V} / \mathrm{V}))$ em leite de jorro cônico-cilíndrico, em termos de eficiência de produção de pó, consumo específico de energia, eficiência térmica e umidade do pó. Constatou-se que o tipo e as características físicas do inerte (poliestireno e polipropileno), a vazão de alimentação e o modo de alimentação da pasta (atomização ou gotejamento), influenciaram os parâmetros de desempenho do processo. Os melhores resultados de produção de pó foram obidos quando se empregou o poliestireno como inerte, a menor vazão de alimentação $(0,12 \mathrm{~kg} / \mathrm{h})$ e o modo de alimentação por gotejamento; entretanto, tais condições resultaram em altos valores de consumo específico de energia. Todavia, a quantidade de energia térmica fornecida ao sistema de secagem pode ser consideravelmente reduzida a partir da desumidificação e recirculação do ar de saída do leito.
\end{abstract}

\section{INTRODUÇÃO}

A secagem de pastas em leito de jorro ocorre na presença de partículas inertes, que agem tanto como um suporte para as pastas, como uma fonte de calor para a secagem. Neste processo, a pasta empregada pode ser atomizada através de um bico atomizador de simples ou duplo fluido ou gotejada sobre o leito móvel de partículas (FREIRE, FERREIRA e FREIRE, 2011; BRAGA e ROCHA, 2013).

Durante o processo de secagem, o leito se torna molhado e a pasta recobre gradativamente a superfície dos inertes formando uma fina camada de material. $\mathrm{O}$ filme sobre a superfície do inerte é seco devido à transferência de calor da própria partícula (condução) e convectivamente a partir do ar quente que entra no leito. $\mathrm{O}$ recobrimento atinge um nível crítico a partir do qual o filme é removido da superfície do inerte, na forma de pó, devido ao atrito entre os mesmos e com a parede do leito (BARRET e FANE, 1990; FREIRE, FERREIRA e FREIRE, 2011; OCHOA-MARTINEZ, BRENNAN e NIRANJAN, 1993; ROCHA e TARANTO, 2008).

A medida em que o leito é alimentado continuamente, os estágios de formação do filme, secagem, fratura do filme e elutriação ocorrem simultaneamente. Na prática, a taxa de produção e remoção do pó deve ser sempre maior ou igual a taxa de alimentação da pasta, evitando a aglomeração e acumulação de material no leito (BARRET e FANE, 1990; FREIRE, FERREIRA e FREIRE, 2011; PHAM, 1983).

A secagem de materiais termo sensíveis em leito de jorro tem sido estudada com foco 
na fluidodinâmica do leito e nos parâmetros operacionais relacionando-os aos parâmetros de eficiência e qualidade do processo e do produto. A qualidade do pó pode ser qualificada pelo sabor, textura e cor e determinada por uma ampla gama de métodos disponíveis na literatura, todavia, está submetida à aceitação dos consumidores (BENALI e AMAZOUZ, 2006).

A secagem pode representar uma significante fração do consumo de energia de um processo, dependendo do tipo de indústria e da tecnologia empregada. Na produção de materiais provenientes da madeira, por exemplo, a energia requerida para a secagem pode chegar a $70 \%$ da energia total do processamento. Além disso, tanto os processos de secagem de commodities e materiais de baixo custo como os processos de secagem de materiais com alto valor agregado, requerem um baixo consumo de energia, evitando a elevação dos custos de produção (KUDRA, 2004). Portanto, o desafio reside na otimização das interações entre a eficiência energética, eficiência de produção e qualidade do produto.

Neste trabalho são apresentados resultados de desempenho do processo de secagem da pasta leite reconstituído-polpa de amora (25\%: 75\% (V/V)) (eficiência de produção de pó), dos aspectos energéticos (consumo específico de energia, eficiência térmica de secagem), e de umidade do pó para diferentes condições operacionais. Para a condição otimizada em relação à eficiência de produção de pó, são apresentadas as curvas de secagem e análise da evolução do processo (eficiência de obtenção de pó e massa de pó coletada em função do tempo de processo).

\section{MATERIAIS E MÉTODOS}

\subsection{Preparação da pasta}

A polpa de amora preta in natura foi obtida a partir de frutas congeladas da marca DeMarchi (Jundiaí, São Paulo), seguindo o procedimento: descongelamento das amoras; trituração das frutas; prensagem empregando filtro de pano; homogeneização; congelamento a $-18{ }^{\circ} \mathrm{C}$. A polpa não foi submetida a tratamento enzimático, sendo diretamente congelada. O período de armazenamento variou de 3 a 11 meses.

Empregou-se leite reconstituído como adjuvante nos processos de secagem da polpa de amora com intuito de aumentar a concentração de sólidos (diminuindo o conteúdo de água a ser evaporado) e melhorar o desempenho do processo (BRAGA e ROCHA, 2013; BRAGA, 2014; BRAGA e ROCHA, 2015). Para tanto, utilizou-se leite em pó da marca Nestlé (Ninho), seguindo o procedimento (para $100 \mathrm{~g}$ de pasta): $50 \mathrm{~g}$ de leite em pó; $50 \mathrm{~g}$ de água; mistura do leite em pó e da água.

A razão volumétrica da pasta empregada na secagem foi de $25 \%$ leite reconstituído: $75 \%$ polpa de amora in natura, tal formulação foi otimizada conforme Braga e Rocha (2014).

\subsection{Caracterização física da pasta}

A pasta leite reconstituído-polpa de amora (25\%: $75 \%, \mathrm{~V} / \mathrm{V})$ foi caracterizada quanto à densidade e umidade. A densidade foi determinada por picnometria, em triplicata. A umidade da pasta foi determinada pelo método da estufa a $105^{\circ} \mathrm{C}$ para secagem do solvente até peso constante, em triplicata.

\subsection{Umidade do pó}

A umidade do pó $\left(X_{\mathrm{pó}}\right)$ foi determinada a partir da secagem de $1 \mathrm{~g}$ das amostras em estufa a vácuo de $20 \mathrm{inHg}$ por 24 horas à 70 ${ }^{\circ} \mathrm{C}$, em duplicata.

\subsection{Sistema experimental e condições fixas de operação}

Os ensaios de secagem foram conduzidos em leito de jorro do tipo cônicocilíndrico construído em acrílico Plexiglas ${ }^{\circledR}$, com as seguintes dimensões: diâmetro do 
leito de $20 \mathrm{~cm}$, altura da coluna cilíndrica de $30 \mathrm{~cm}$, altura da base cônica de $14 \mathrm{~cm}$, diâmetro do orifício de entrada do ar de $3 \mathrm{~cm}$ e ângulo de inclinação da base cônica de $60^{\circ}$. $\mathrm{Na}$ parte superior do leito de jorro foi conectado um ciclone Lapple em aço inox.

A Figura (1) apresenta o esquema do sistema experimental. O processo de secagem foi monitorado através do uso de instrumentos como termohigrômetros (Cole Parmer, com faixa de medida de 0,5 a $100 \%$ para umidade relativa e temperatura de 10 a $90{ }^{\circ} \mathrm{C}$, precisão de $\pm 1,5$ e $\pm 0,2^{\circ}$, respectivamente), e termopar de cobre-constantan ligado ao controlador de temperatura.

Figura 1 - Esquema experimental

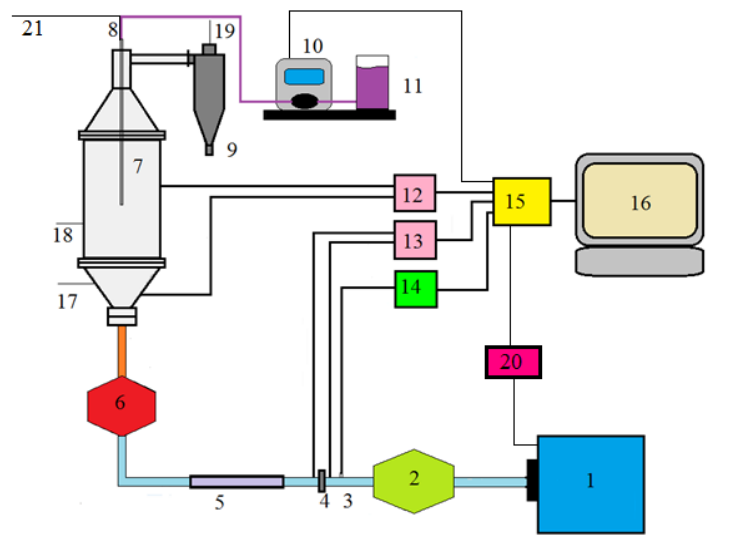

Esquema experimental: (1) soprador; (2) trocador de calor; (3) tomada de pressão estática; (4) tomada de pressão na placa de orifício; (5) leito de sílica gel; (6) aquecedor; (7) leite de jorro cônico-cilíndrico; (8) linha de alimentação da pasta; (9) ciclone Lapple; (10) bomba peristáltica; (11) pasta; (12 e 13) transdutores diferenciais; (14) transdutor absoluto; (15) sistema de aquisição de dados; (16) microcomputador; (17, 18 e 19) termohigrômetros; (20) inversor de frequência; (21) linha de ar comprimido.

Antecedendo este trabalho, realizaramse caracterizações físicas dos inertes polipropileno PP (forma irregular) e poliestireno PS (forma lenticular) (diâmetro de Sauter $\left(d_{\mathrm{p}}\right)$, densidade real $\left(\rho_{\mathrm{r}}\right)$ e esfericidade $(\Phi)$ ), análises fluidodinâmicas (massa de inertes $(M)$, vazão de jorro mínima $\left(W_{\mathrm{jm}}\right)$, vazão de ar de secagem $\left(W_{\mathrm{ar}}\right)$, e queda de pressão máxima $\left(\Delta \mathrm{P}_{\max }\right)$ ), além de testes preliminares de secagem. A partir dos resultados obtidos, estabeleceram-se as condições fixas de operação apresentadas na Tabela 1 (BRAGA e ROCHA, 2013).

Tabela 1 - Caracterizações físicas, parâmetros fluidodinâmicos e condições operacionais fixas empregadas nos processos de secagem.

\begin{tabular}{lcccc}
\hline Inerte & PS & Des. & PP & Des. \\
\hline$d_{\mathrm{p}}(\mathrm{mm})^{\mathrm{a}}$ & 5,19 & 0,26 & 3,14 & 0,18 \\
$\rho_{\mathrm{r}}\left(\mathrm{kg} / \mathrm{m}^{3}\right)^{\mathrm{b}}$ & 1060,0 & 0,6 & 907,8 & 0,5 \\
$\Phi^{\mathrm{a}}$ & 0,90 & 0,07 & 0,74 & 0,06 \\
$M(\mathrm{~kg})$ & 1,4 & - & 1,4 & - \\
$W_{\mathrm{jm}}(\mathrm{kg} / \mathrm{min})^{\mathrm{b}}$ & 0,79 & 0,00 & 0,87 & 0,01 \\
$\Delta \mathrm{P}_{\max }(\mathrm{Pa})^{\mathrm{b}}$ & 1813 & 23 & 1371 & 18 \\
$W_{\mathrm{ar}}(\mathrm{kg} / \mathrm{min})$ & $1,2 . W_{\mathrm{jm}}$ & - & $1,2 . W_{\mathrm{jm}}$ & - \\
\hline
\end{tabular}

*Des.= desvio: ${ }^{a}$ desvio padrão; ${ }^{\mathrm{b}}$ desvio médio

A partir de testes preliminares, fixou-se a alimentação em $300 \mathrm{~mL}$ de pasta por Ensaio com o objetivo de operar em regime permanente de obtenção de pó, atenuando assim, a influência da variação inicial da obtenção de material sólido adicionado ao leito.

\subsection{Ensaios de secagem}

Os processos de secagem foram iniciados somente quando o sistema entrou em regime permanente de temperatura do ar de entrada e saída. Após o termino da alimentação da pasta $(300 \mathrm{~mL})$, a secagem foi prolongada por mais $20 \mathrm{~min}$ com o objetivo de coletar o pó residual desprendido do leito e dos inertes. Para vários ensaios, uma grande quantidade de material foi coletada após o fim da alimentação da pasta, sendo indispensável 
considerar esse período para o cálculo de eficiência de produção de pó.

O polipropileno foi escolhido como o inerte para iniciar a investigação da influência de três variáveis operacionais sobre a eficiência de produção de pó: temperatura do ar de entrada $\left(T_{2}\right)$; pressão de atomização $\left(P_{\mathrm{at}}\right)$; vazão de alimentação da pasta $\left(W_{\mathrm{f}}\right)$. Para tanto, uma matriz de planejamento $2^{3-1}$, com ponto central foi construída.

A partir do trabalho de Medeiros (2010) e da melhor condição operacional encontrada em termos de eficiência de produção, estudou-se o processo de secagem com alimentação da pasta por gotejamento $(\mathrm{G})$, empregando como inerte o PP e o PS.

Para uma conclusiva análise da influência do tipo de alimentação (por gotejamento ou atomização) sobre o desempenho do processo, dois ensaios foram realizados empregando o PS e as condições operacionais: $T_{2}=60{ }^{\circ} \mathrm{C} ; W_{\mathrm{f}}=2 \mathrm{~mL} / \mathrm{min} ; P_{\mathrm{at}}=$ 10 psig (BRAGA e ROCHA, 2013).

Com os dados de temperatura de bulbo seco e umidade relativa de entrada (2) e saída (3) do leito, obtidos durante a realização dos ensaios de secagem, realizou-se a análise energética dos processos de secagem da pasta leite reconstituído-polpa de amora.

A eficiência da produção de pó $(\xi)$ foi calculada através da razão entre a massa coletada no ciclone $\left(M_{\text {pó}}\right)$ pela massa total de sólidos adicionada ao leito $\left(M_{\text {pasta }}\right)$, em base seca, Equação (1).

$\xi=\left(M_{\text {pó }} / M_{\text {pasta }}\right) .100$

\subsection{Aspectos energéticos}

O consumo de energia, avaliado neste trabalho, compreende o calor requerido para o aquecimento dos inertes e da pasta à temperatura de processo e a compensação devido à perda de calor na linha de secagem. A Figura 2 apresenta o esquema da passagem do ar e da pasta através da linha de secagem.
Figura 2 - Esquema da passagem do ar e da pasta através da linha de secagem.

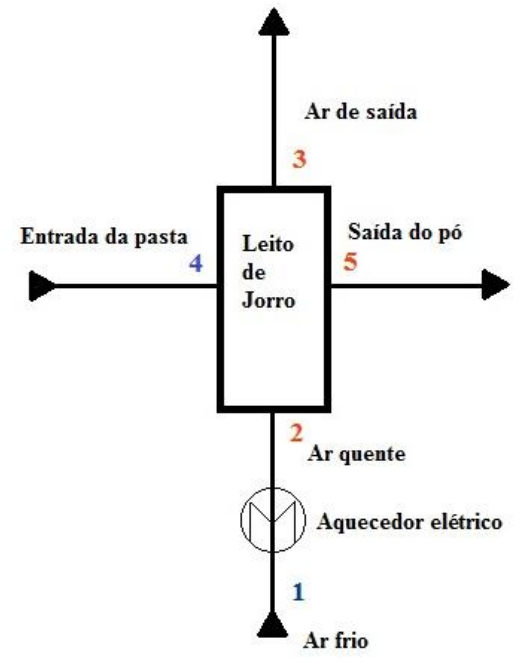

Fonte: Adaptado de BENALI e AMAZOUZ (2006).

Do ponto de vista industrial, o parâmetro energético determinante é o consumo específico de energia $\left(\varepsilon_{\mathrm{sp}}\right)$ de um sistema de secagem para um dado processo (BENALI e AMAZOUZ, 2006). Por sua vez, tal parâmetro está relacionado à variação da entalpia do ar, Equação (2).

$h_{\mathrm{ar}}=\left(C_{\mathrm{pg}}+Y \cdot C_{\mathrm{pv}}\right) \cdot T+Y \cdot \lambda_{\mathrm{v}}$

A energia requerida $\left(q_{\mathrm{r}}\right)$ para alcançar o conteúdo final de umidade é determinada a partir da Equação (3).

$q_{\mathrm{r}}=W_{\mathrm{as}} \cdot\left[\left(h_{\mathrm{ar}}\right)_{2}-\left(h_{\mathrm{ar}}\right)_{1}\right]$

O consumo específico de energia é definido com a razão entre a energia transferida ao sistema de secagem e a massa de água evaporada, Equação (4).

$\varepsilon_{\mathrm{sp}}=\mathrm{q}_{\mathrm{r}} /\left(W_{\mathrm{f} .}\left(X_{\mathrm{pasta}}-X_{\mathrm{pó}} / 1-X_{\mathrm{pó}}\right)\right)$

$\mathrm{Na}$ secagem convectiva, em processos adiabáticos, para baixos valores de umidade e temperatura e capacidades caloríficas constantes, a eficiência energética pode ser 
estimada a partir da eficiência térmica de

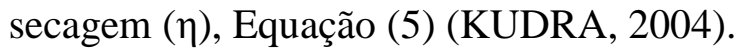

$\eta=\left[(T)_{2}-(T)_{3}\right] /\left[(T)_{2}-\left(T_{\text {bu }}\right)\right] .100$

\subsection{Curvas de secagem}

As curvas de secagem foram construídas a partir da metodologia empregada por Rocha et al. (2011). O procedimento para obtenção das taxas de evaporação e das curvas de secagem consistiu em: após o sistema entrar em regime permanente de temperatura, registraram-se as respectivas medidas de umidade relativa $\mathrm{e}$ temperaturas de bulbo seco do ar na entrada e saída do leito. Para determinação das demais propriedades do ar, utilizaram-se relações psicrométricas. A taxa de evaporação ( $\left.W_{\text {evp }}\right)$ foi calculada através da Equação (6).

$W_{\text {evp }}=W_{\text {as }} \cdot\left(Y_{3}-Y_{2}\right)$

\section{RESULTADOS}

\subsection{Caracterização física da pasta}

A pasta composta por $25 \%$ de leite reconstituído e $75 \%$ de polpa de amora $(\mathrm{V} / \mathrm{V})$, ou $25,3 \%$ de leite reconstituído e $74,7 \%$ de polpa de amora $(\mathrm{m} / \mathrm{m})$ apresentou densidade de 1046,9 com desvio médio de $0,5 \mathrm{~kg} / \mathrm{m}^{3}$ e umidade de aproximadamente $83,3 \%$ (concentração de sólidos de 0,167 com desvio médio de $0,002 \mathrm{~kg} / \mathrm{kg}$ ).

\subsection{Produção de pó e aspectos energéticos}

A Tabela 2 apresenta os valores de consumo específico de energia, eficiência térmica, umidade do pó e eficiência de produção de pó, usando como inerte o PP e diferentes condições operacionais.

Com exceção dos experimentos 1 e 2, todos os Ensaios apresentaram valores de eficiência de produção de pó abaixo de $30 \%$, sendo que grande parte do material solido permaneceu retido sobre a superfície dos inertes, paredes do leito e ciclone ou foi perdido na forma de finos. Constatou-se que a eficiência aumentou com o decréscimo da vazão de alimentação da pasta. Para a vazão de $0,12 \mathrm{~kg} / \mathrm{h}$ (maior eficiência), observou-se o efeito negativo do aumento da temperatura, Ensaios 3 e 4, sem influência significativa da pressão de atomização, Ensaios 1 e 2.

Tabela 2 - Consumo específico de energia, eficiência térmica, umidade do pó e eficiência de produção de pó, usando como inerte o PP.

\begin{tabular}{|c|c|c|c|c|c|}
\hline Ensaio & $\begin{array}{c}\text { Condições } \\
\text { operacionais } \\
\left(W_{\mathrm{f}}(\mathrm{kg} / \mathrm{h}),\right. \\
T_{2}\left({ }^{\circ} \mathrm{C}\right), P_{\mathrm{at}} \\
(\mathrm{psig}))\end{array}$ & $\begin{array}{c}\varepsilon_{\mathrm{sp}} \\
(\mathrm{MJ} / \mathrm{kg})\end{array}$ & $\begin{array}{c}\eta \\
(\%)\end{array}$ & $\begin{array}{c}X_{\mathrm{p} 0} \\
(\%)^{*}\end{array}$ & $\begin{array}{c}\xi \\
(\%)^{*}\end{array}$ \\
\hline 1 & $\begin{array}{c}0,12,60 \\
10\end{array}$ & 12,6 & 16,5 & 3,50 & 33,8 \\
\hline 2 & $\begin{array}{c}0,12,60 \\
20\end{array}$ & 13,0 & 19,8 & 2,60 & 33,3 \\
\hline 3 & $\begin{array}{c}0,12,80 \\
10\end{array}$ & - & 19,6 & 1,60 & 23,3 \\
\hline 4 & $\begin{array}{c}0,12,80 \\
20\end{array}$ & - & 22,8 & 0,92 & 26,5 \\
\hline 5 & $\begin{array}{c}0,25,60 \\
10\end{array}$ & 5,7 & 24,8 & 3,00 & 21,1 \\
\hline 6 & $\begin{array}{c}0,25,60 \\
20\end{array}$ & 4,1 & 27,9 & 3,12 & 8,9 \\
\hline 7 & $\begin{array}{c}0,25,80 \\
10\end{array}$ & - & 25,1 & 3,10 & 9,7 \\
\hline 8 & $\begin{array}{c}0,25,80 \\
20\end{array}$ & - & 23,6 & 2,60 & 14,4 \\
\hline 9 & $\begin{array}{c}0,18,70 \\
15\end{array}$ & - & 20,4 & 2,10 & 22,1 \\
\hline 10 & $\begin{array}{c}0,18,70 \\
15\end{array}$ & - & 18,5 & 2,66 & 19,2 \\
\hline 11 & $\begin{array}{c}0,18,70, \\
15\end{array}$ & - & 20,5 & 2,13 & 20,4 \\
\hline
\end{tabular}

${ }^{*}$ Resultados obtidos em Braga e Rocha (2013)

Devido aos baixos valores de umidade relativa obtidos durante os processos de secagem onde se empregou $T_{2}$ de 70 e $80{ }^{\circ} \mathrm{C}$, uma vez que a precisão do termohigrômetro era de $\pm 1,5 \%$, optou-se por não calcular os valores do consumo específico de energia. Portanto, tal parâmetro foi estimado apenas para $T_{2}$ de $60{ }^{\circ} \mathrm{C}$, Tabela 2. Como esperado, constatou-se que o consumo específico de energia diminuiu com o aumento da vazão de alimentação da pasta, atingindo um valor na 
ordem de 4,1 MJ por kg de água evaporada, Ensaio 6.

Benali e Amazouz (2006) analisaram o consumo específico de energia do processo de secagem de pastas de amido vegetal em leito de jorro cônico diluído (Jet spouted bed), empregando como inertes partículas cúbicas de Teflon ${ }^{\circledR}$ (condutividade térmica de 0,202 $\mathrm{W} / \mathrm{mK}$ (YANG, 2007)). A alimentação das pastas se deu por atomização, a temperatura do ar de entrada variou de $140{ }^{\circ} \mathrm{C}$ a $240{ }^{\circ} \mathrm{C}$, utilizou-se $12 \mathrm{~kg}$ de inertes e vazão de alimentação de $107,5 \mathrm{~kg} / \mathrm{h}$. Constatou-se que o consumo específico de energia aumentou com o aumento da diferença da temperatura de entrada e saída do leito e da concentração de sólidos das pastas, variando de 3,8 MJ/kg água evaporada à $5,3 \mathrm{MJ} / \mathrm{kg}$ água evaporada.

Wachiraphansakul e Devahastin (2005) estimaram o consumo específico de energia do processo de secagem de Okara, também em leito de jorro diluído. O menor valor de $\varepsilon_{\mathrm{sp}}$ $(3,69 \mathrm{MJ} / \mathrm{kg}$ água) foi obtido quando se empregou os maiores níveis das condições operacionais avaliadas: velocidade superfícial do ar de $1,5 \mathrm{~m} / \mathrm{s}$; altura do leito de Okara de $18 \mathrm{~cm} ; T_{2}$ de $130{ }^{\circ} \mathrm{C}$. Apesar deste conjunto de condições operacionais requerer uma maior quantidade de energia para aquecer o ar de jorro, a combinação de um menor tempo para a secagem e de uma maior capacidade de evaporação da água, resultou nos menores valores de consumo específico de energia.

Medeiros (2010) estudou a secagem de leite de cabra em leito de jorro cônicocilíndrico. As condições operacionais analisados com o intuito de maximizar a obtenção de pó foram: tipo de inerte (PP ou PEAD); tipo de alimentação do leite de cabra (gotejante ou atomizado); forma de alimentação do leite de cabra (contínuo ou intermitente). Os resultados de eficiência de produção de pó variaram de 2,4 a $64,4 \%$, sendo a melhor condição obtida quando se empregou: inerte PP; alimentação gotejante; processo contínuo.
A partir do trabalho de Medeiros (2010) e da melhor condição operacional encontrada em termos de eficiência de produção de pó, Tabela 2 Ensaio $1\left(T_{2}=60^{\circ} \mathrm{C}\right.$ e $W_{\mathrm{f}}=0,12$ $\mathrm{kg} / \mathrm{h}$ ), estudou-se o processo de secagem com alimentação da pasta por gotejamento e empregando como inerte o PP e o PS. O intuito desta etapa do estudo foi definir o inerte e a condição operacional apropriada para a secagem da pasta leite-polpa de amora (25\%: $75 \%(\mathrm{~V} / \mathrm{V}))$, resultando em uma maior eficiência de produção de pó com baixo teor de umidade. A Tabela 3 apresenta os valores de consumo específico de energia, eficiência térmica, umidade do pó e eficiência de produção de pó, Ensaios em duplicata.

Para o PP, Ensaios 12 e 13, observou-se um aumento da eficiência de produção de pó de aproximadamente $24,8 \%$ em relação ao processo usando atomização, Tabela 2 Ensaio 1. Entretanto, a eficiência média permaneceu abaixo de $60 \%$, o que dificilmente resultaria em um processo viável.

Os resultados empregando como inerte o PS, Ensaios 14 e 15, apresentaram uma boa eficiência de processo $\left(\xi_{\text {média }}=63,27 \%\right)$, na mesma ordem de grandeza da obtida por Medeiros (2010). Tal resultado se deve às características físicas dos inertes, superfície lisa e uniforme, reduzindo as zonas de acúmulo e estagnação de pasta. Durante os ensaios, visualizou-se grande produção de pó e uma tênue diminuição na velocidade de circulação dos inertes. Concluiu-se, portanto, que o melhor inerte a ser empregado nos ensaios de secagem da pasta leite reconstituído-polpa amora foi o PS.

Para uma conclusiva análise da influência do tipo de alimentação (por gotejamento ou atomização) no desempenho do processo, dois ensaios foram realizados empregando partículas de PS $\left(T_{2}=60^{\circ} \mathrm{C}, W_{\mathrm{f}}=\right.$ $0,12 \mathrm{~kg} / \mathrm{h}$ e $P_{\mathrm{at}}=10 \mathrm{psig}$ ). Verificou-se uma maior eficiência para o processo com alimentação gotejante, aproximadamente $12,37 \%$, quando comparado ao processo por 
atomização. Visualmente constatou-se a formação de uma película sobre a superfície dos inertes quando se empregou a atomização da pasta, fato também verificado para as partículas de PP e que resultou em maior retenção de material no leito.

Analisando a Tabela 3, constatou-se que o consumo específico de energia aumentou consideravelmente quando se empregou como inerte o PS e a alimentação da pasta por gotejamento. Tal fato pode estar relacionado às caracteristicas físicas do PS, dentre elas a condutividade térmica $(\mathrm{k}=0,105 \mathrm{~W} / \mathrm{mK}$; sendo para o $\mathrm{PP}, \mathrm{k}=0,12 \mathrm{~W} / \mathrm{mK}$ (YANG, 2007)) e, também, a maior resistência à evaporação da água presente nas gotas gotejadas devido à menor área superfícial das mesmas, quando comparada à das gotículas formadas durante a atomização da pasta.

Tabela 3 - Consumo de energia específica, eficiência térmica, umidade do pó e eficiência de produção de pó. Condições operacionais fixas: $W_{\mathrm{f}}=0,12 \mathrm{~kg} / \mathrm{h} ; T_{2}=60^{\circ} \mathrm{C}$.

\begin{tabular}{cccccc}
\hline Ensaio & $\begin{array}{c}\text { Alim. } \\
\text { linerte }\end{array}$ & $\begin{array}{c}\varepsilon_{\mathrm{sp}} \\
(\mathrm{MJ} / \mathrm{kg})\end{array}$ & $\begin{array}{c}\eta \\
(\%)\end{array}$ & $\begin{array}{c}X_{\mathrm{p} \sigma} \\
(\%)^{*}\end{array}$ & $\begin{array}{c}\xi \\
(\%)^{*}\end{array}$ \\
\hline 12 & $\mathrm{G} / \mathrm{PP}$ & 21,7 & 21,7 & 1,12 & 45,3 \\
13 & $\mathrm{G} / \mathrm{PP}$ & - & - & & \\
14 & $\mathrm{G} / \mathrm{PS}$ & 19,0 & 26,2 & 3,43 & 63,8 \\
15 & $\mathrm{G} / \mathrm{PS}$ & 21,5 & 22,3 & 2,74 & 62,7 \\
16 & $10 \mathrm{psig} / \mathrm{PS}$ & 17,6 & 31,8 & 2,50 & 53,3 \\
17 & $10 \mathrm{psig} / \mathrm{PS}$ & 16,6 & 31,0 & 2,97 & 55,5 \\
\hline
\end{tabular}

${ }^{*}$ Resultados obtidos em Braga e Rocha (2013)

A eficiência térmica do processo de secagem se manteve abaixo de $32 \%$, para todos os Ensaios. Os baixos valores de eficiência estão diretamente relacionados à pequena diferença de temperatura do ar de entrada e saída do leito $\left(T_{2}-T_{3}\right)$. Por exemplo, analisando a Figura 3 Ensaios 14 e 15, esta diferença foi inferior a $10{ }^{\circ} \mathrm{C}$. Todavia, o decréscimo da temperatura do ar de saída deve ser analisado com critério, pois resulta no aumento da umidade do pó, o que pode comprometer a qualidade do produto.

No entanto, a quantidade de energia térmica fornecida ao sistema pode ser consideravelmente reduzida. $\mathrm{O}$ ar de saída do leito apresentou pequena quantidade de umidade e temperatura elevada, pondendo ser desumidificado e recirculado. Por exemplo, se o ar de saída do leito, Ensaio 15, fosse desumidificado até atingir a umidade absoluta do ar de entrada do primeiro ciclo, à uma temperatura $T_{1}=50^{\circ} \mathrm{C}$, o consumo específico de energia diminuiria para aproximadamente 4,3 $\mathrm{MJ} / \mathrm{kg}$ água. $\mathrm{O}$ procedimento sugerido redúz o consumo líquido de energia $e$, consequentemente, acarreta em um menor valor do consumo específico de energia, conforme reporta a literatura (JUMAH e MUJUMDAR, 1996; WACHIRAPHANSAKUL e DEVAHASTIN, 2005).

A Figura 3 apresenta a diferença de temperatura do ar de entrada e saída do leito $\left(T_{2}-T_{3}\right)$ durante os processos de secagem da pasta leite reconstituído-polpa de amora (25\%: 75\% (V/V)), Ensaios 14 e 15. Observou-se que a diferença de temperatura se manteve praticamente constante a partir dos 5 minutos de processo.

Figura 3 - Diferença de temperatura do ar de entrada e saída do leito em função do tempo de secagem $(\circ)$ Ensaio 14 e $(\Delta)$ Ensaio 15.

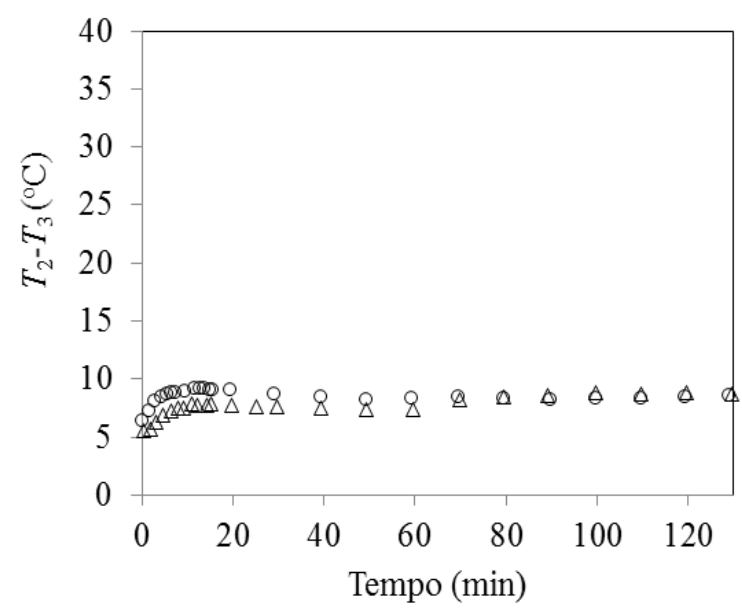




\subsection{Umidade do pó}

Analisando as Tabelas 2 e 3, observouse que os valores de umidade dos pós permaneceram abaixo de 3,55\%. Usando o leite como parâmetro de comparação, a umidade máxima deve ser de $4 \%$ para o leite em pó desnatado e de 2,5\% para o leite em pó integral, estando o excesso de umidade relacionado à rápida perda de sabor, solubilidade e alterações nas propriedades físicas do produto (ANVISA, 2008).

\subsection{Evolução da eficiência de produção de pó e massa de sólidos acumulada}

A Figura 4 apresenta os valores de massa seca de pó coletada em função do tempo de processo para a pasta leite-polpa de amora (25\%: 75\% (V/V)). Do início da secagem até aproximadamente $50 \mathrm{~min}$, observou-se um crescimento linear da obtenção de pó, e a partir deste ponto, o processo entrou em um estado praticamente estacionário de produção.

Figura 4 - Obtenção de pó em função do tempo (०) Ensaio 14 e $(\Delta)$ Ensaio 15.

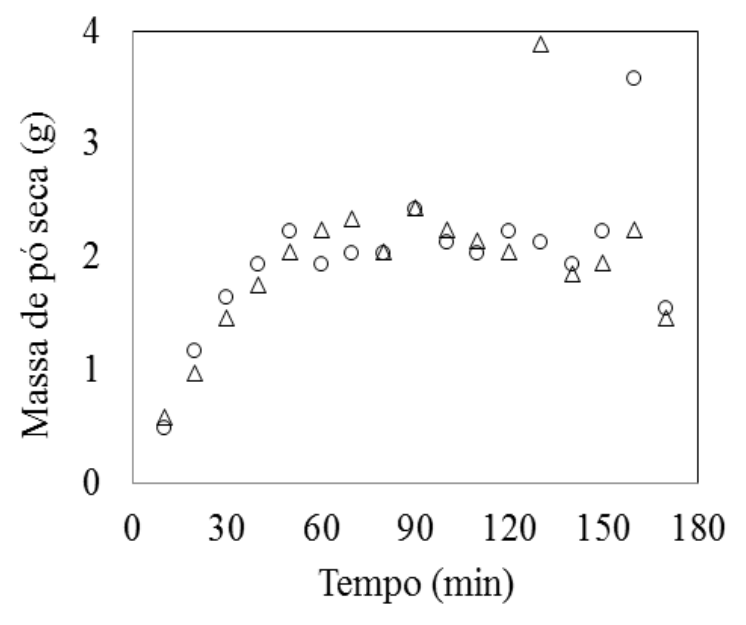

Analisando a eficiência da obtenção de pó em função do tempo para a pasta leitepolpa de amora (25\%: 75\% (V/V)), Figura 5, constatou-se que tal parâmetro aumentou com o tempo de processo, apresentando três períodos distintos de crescimento: de 0 a 50 min (aumento linear acentuado); de 50 a 150 min (tênue aumento da eficiência de produção de pó); 150 a 170 min (observa-se um acentuado aumento da eficiência de produção de pó). Torna-se importante destacar a influência do período pós-alimentação (20 min) na eficiência de produção de pó ( $\xi_{\text {média }}=$ $54,4 \%$ em $t=150$ min; e $\xi_{\text {média }}=63,2 \%$ em $t=$ $170 \mathrm{~min}$ ), aumento de $16,2 \%$.

Figura 5 - Eficiência da obtenção de pó em função do tempo (o) Ensaio 14 e $(\Delta)$ Ensaio 15.

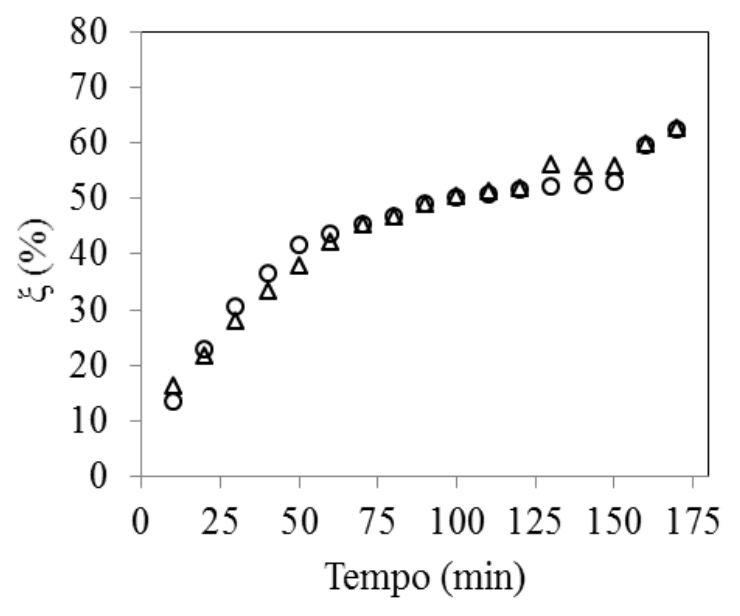

\subsection{Curvas de secagem}

A Figura 6 apresenta a diferença de umidade absoluta do ar de entrada e saída do leito $\left(Y_{3}-Y_{2}\right)(\mathrm{A})$, e a taxa de evaporação durante o processo de secagem da pasta (B). Constatou-se que a evaporação ocorreu rapidamente, sendo que já nos instantes iniciais do processo a diferença de umidade absoluta do ar e a taxa de evaporação atingiram valores praticamente constante. Tal comportamento também é reportado na literatura sobre secagem de pastas (BRAGA et al., 2015; MEDEIROS, 2010).

As temperaturas do ar de entrada $\left(T_{2}\right) \mathrm{e}$ de saída $\left(T_{3}\right)$ e a umidade relativa do ar de saída do leito foram monitoradas durante a realização dos ensaios de secagem. A umidade relativa do ar de entrada foi determinada antes do início da alimentação da pasta. Como resultado geral, a diferença máxima de temperatura $\left(T_{2}-T_{3}\right)$ foi de $12,5{ }^{\circ} \mathrm{C}$ 
e a máxima umidade relativa de entrada foi de $8,7 \%$. Um menor valor de umidade absoluta foi assegurado devido à passagem do ar pelo leito de sílica gel.

Figura 6 - Diferença de umidade absoluta do ar (A) e taxa de evaporação durante o processo de secagem (B): (०) Ensaio $14\left(Y_{2}=0,0094 \mathrm{~kg}\right.$ água $/ \mathrm{kg}$ ar seco $)$ e $(\Delta)$ Ensaio $15\left(Y_{2}=0,0067 \mathrm{~kg}\right.$ água/kg ar seco).

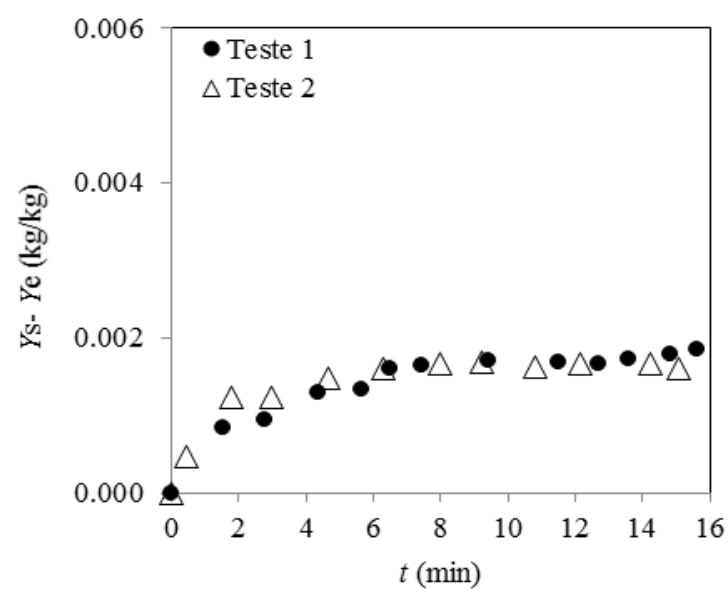

(A)

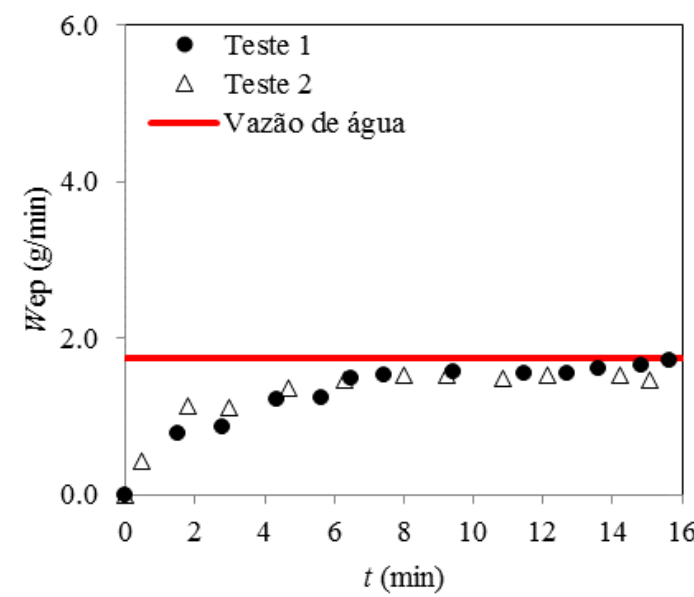

(B)

\section{CONCLUSÕES}

A utilização do poliestireno como inerte, alimentação da pasta por gotejamento e a menor vazão de alimentação utilizada, resultou em boa eficiência de produção de pó, na ordem de $63 \%$. Em contra partida, tais condições resultaram em altos valores de consumo específico de energia.

A eficiência térmica do processo de secagem se manteve abaixo de $32 \%$, para todos os ensaios. Os baixos valores de eficiência se devem à pequena diferença de temperatura do ar de entrada e saída do leito.

Constatou-se que a evaporação ocorreu rapidamente, sendo que já nos instantes iniciais do processo a diferença de umidade absoluta do ar de entrada e saída do leito atingiu um valor praticamente constante.

A quantidade de energia térmica fornecida ao sistema de secagem pode ser consideravelmente reduzida a partir da desumidificação e recirculação do ar de saída do leito.

\section{NOMENCLATURA}

$C_{\mathrm{pg}} \quad$ calor específico do gás $\quad(\mathrm{J} / \mathrm{kgK})$

$C_{\mathrm{pv}} \quad$ calor específico do vapor $\quad(\mathrm{J} / \mathrm{kgK})$

$d_{p} \quad$ diâmetro médio dos inertes (mm)

$h_{\mathrm{ar}} \quad$ entalpia do ar $\mathrm{J} / \mathrm{kg}$ ar seco

$M \quad$ massa de inertes $\quad(\mathrm{kg})$

$M_{\text {pasta }} \quad$ massa de sólidos adicionada $(\mathrm{kg})$

$M_{\text {pó }} \quad$ massa seca de pó coletada $\quad(\mathrm{kg})$

$P_{a t} \quad$ pressão de atomização (psig)

PEAD polietileno de alta densidade (-)

PP polipropileno (-)

PS poliestireno (-)

$q_{\mathrm{r}} \quad$ energia requerida $\quad(\mathrm{J} / \mathrm{s})$

$T$ temperatura $\quad\left({ }^{\circ} \mathrm{C}\right)$

$T_{\text {bu }} \quad$ temperatura de bulbo úmido $\quad\left({ }^{\circ} \mathrm{C}\right)$

$X_{\text {pasta }} \quad$ umidade da pasta $\quad(\mathrm{kg} / \mathrm{kg})$ b.u.

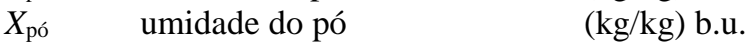

$W_{a r} \quad$ vazão do ar de secagem (kg/min)

$W_{\text {as }} \quad$ vazão mássica de ar seco $\quad(\mathrm{kg} / \mathrm{s})$

$W_{\mathrm{f}} \quad$ vazão de alimentação $\quad(\mathrm{kg} / \mathrm{s})$

$W_{\text {jm }} \quad$ vazão de jorro mínimo (kg/min)

$Y \quad$ umidade absoluta do ar (kg água/kg

ar seco)

$\Phi \quad$ esfericidade dos inertes (-)

$\xi \quad$ eficiência da produção de pó $\quad(\%)$

$\eta \quad$ eficiência térmica $\quad(\%)$

$\lambda_{\mathrm{v}} \quad$ calor latente de vaporização $\quad(\mathrm{J} / \mathrm{kg})$

$\rho_{\text {real }} \quad$ densidade real $\left(\mathrm{kg} / \mathrm{m}^{3}\right)$

$\Delta \mathrm{P}_{\max } \quad$ queda de pressão máxima $\quad(\mathrm{Pa})$ 


\section{REFERENCIAS}

BARRET, N.; FANE, A. Drying liquid materials in spouted bed. In: Mujundar, A. S. e Roques, M. A. (org). Drying' 89. New York: Hemisphere Publishing Corp., 1990, p. 426-432.

BENALI, M.; AMAZOUZ, M. Drying of vegetable starch solutions on inert particles quality and energy aspects. Journal of Food Engineering. v. 76, p. 484-489, 2005.

BRAGA, M. B.; ROCHA, S. C. S. Drying of milk-blackberry pulp mixture in spouted bed. Canadian Journal of Chemical Engineering. v. 91, p. 1786-1792, 2013.

BRAGA, M. B. B. Obtenção de mistura leiteamora preta em pó por secagem em leito de jorro. 2014. 187p. Tese (Doutorado em Engenharia Química) - Faculdade de Engenharia Química, Universidade Estadual de Campinas, 2014.

BRAGA, M. B.; ROCHA, S. C. S. Spouted bed drying of milk-blackberry pulp: analysis of powder production efficiency and powder characterization. Drying Technology. v.33, p. 933-940, 2015.

BRAGA, M. B.; WANG, Z.; GRACE, J. R.; JIM LIM, C.; ROCHA, S. C. S. Slot-Rectangular spouted bed: hydrodynamic stability and effects of operating conditions on drying performance. Drying Technology. v.33, p. 216-226, 2015.

FREIRE, J. T.; FERREIRA, M. C.; FREIRE, F. B. Drying of solutions, slurries, and pastes. In: Epstein, N.; Grace, J. R. (org). Spouted and Spout-Fluid Beds. Cambridge: Cambridge University Press, 2011. p.206-221.

JUMAH, R. Y.; MUJUMDAR, A. S. Batch drying kinetics of corn in a novel rotating jet spouted bed. Canadian Journal of Chemical Engineering. p. 479-486, 1996.

KUDRA, T. Energy aspects in drying. Drying Technology. v.22, n.5, p. 917-932, 2004.
MEDEIROS, U. K. L. Viabilidade técnica de uma rota não convencional para a produção de leite de cabra em pó em cooperativas do Rio Grande do Norte. 2010. 166p. Tese (Doutorado em Engenharia Química) - Centro de Tecnologia, Universidade Federal do Rio Grande do Norte, 2010.

OCHOA-MARTINEZ, L. A.; BRENNAN, J. G.; NIRANJAN, K. Spouted bed dryer for liquid foods. Food Control. v. 4, p. 41-45, 1993.

PHAM, Q. T. Behaviour of a conical spouted-bed dryer for animal blood. The Canadian Journal of Chemical Engineering. v. 61, p. 426-434, 1983.

ROCHA, S. C. S.; TARANTO, O. P. Advances in spouted bed drying of foods. In: Cristina Ratti. (org). Advances in Food Dehydration. 1 ed. Boca Raton: Taylor e Francis-CRC press, 2008, v. 5, p.153-186.

ROCHA, S. C. S.; SOUZA, J. S.; ALSINA, O. L. S.; MEDEIROS, M. F. D. Drying of tropical fruit pulps: spouted bed process optimization as a function of pulp composition. Drying Technology. v. 29, p.1587-1599, 2011.

YANG, Y. Thermal conductivity. In: Mark, J. E. (org). Physical properties of polymers handbook. New York: Springer science + Business media, 2007, p. 156-162.

WACHIRAPHANSAKUL, S.; DEVAHASTIN, S. Drying kinetics and quality of soy residue (Okara) dried in a jet spouted-bed dryer. Drying Technology. v. 23, p.1229-1242, 2005.

\section{AGRADECIMENTOS}

Os autores agradecem a FAPESP- Fundação de Amparo à Pesquisa do Estado de São Paulo, pelo apoio financeiro e institucional. 